\section{Surveillance of sexually transmitted infections in primary care}

\section{Simms, A-K Hurtig, P A Rogers, G Hughes, K A Fenton}

What do we need to do?

S urveillance of sexually transmitted infections (STI) provides information for public health action, a relation that was highlighted by the "National Strategy for Sexual Health and HIV" published recently by the Department of Health (England). ${ }^{1}$ Systematically collected, timely, accurate, detailed, representative STI surveillance data are needed to estimate the population burden of disease; monitor effectiveness of STI prevention; evaluate healthcare access; and assess determinants of transmission (box l).

England has one of the world's most comprehensive STI surveillance systems based on data from genitourinary medicine (GUM) clinics (the KC60 return) and laboratory reports. Data are widely disseminated and used in strategic health planning at national and local levels. ${ }^{1}$ A weakness in English surveillance is that it does not provide the information needed to interpret changes in STI epidemiology. This is not unusual as there are similar weaknesses in surveillance systems in other countries, such as Sweden. ${ }^{2}$ Both the KC60 and laboratory report systems are being redeveloped: patient based data collection is being introduced in GUM; enhanced surveillance programmes have

\section{Box 1 Key objectives of STI surveillance}

- detect trends

- provide population estimates of prevalence/incidence of infection/ disease

- identify risk factors associated with infection/disease

- allow assessment of intervention strategies

- provide information to inform clinical practice and public health action

- be of high sensitivity (good coverage of the target conditions)

- use consistent, accurate diagnostic methods

- be able to detect outbreaks

- be effective and efficient

- present and disseminate data in a timely and accessible manner been developed for syphilis and gonorrhoea; and the laboratory report system is being expanded. Although improvements in coverage, availability, quality, timeliness, and representativeness are anticipated, lack of data from primary care remains. ${ }^{3}$ Substantial reservoirs of predominantly asymptomatic STIs, such as Chlamydia trachomatis, genital herpes simplex virus infection (HSV), human papillomavirus infection, and pelvic inflammatory disease (PID) are known to be treated in primary care settings, including general practice and family planning. ${ }^{5-7}$ Developments proposed in the national strategy suggest that STI diagnosis and management will be increasingly focused on primary care. The systematic collection of timely, representative data from primary care is needed if we are to address future prevention needs, an emerging challenge that requires novel approaches to surveillance. Here, we discuss a new method for estimating STI prevalence in primary care within the context of the available surveillance data.

\section{AVAILABLE EVIDENCE AND REASONS FOR OTHER STUDIES}

Population based studies provide the best method of assessing the epidemiology of STIs that are not generally confined to core groups, but such studies are rare. In the United Kingdom, the only investigation of this type was the second National Survey of Sexual Attitudes and Lifestyles (NATSAL 2000), which explored the reported population experience of STI infection, and the prevalence of $C$ trachomatis. ${ }^{7}$ This showed that $69 \%$ of women diagnosed with $C$ trachomatis had attended GUM services, whereas only $30 \%$ diagnosed with PID had attended GUM services. This emphasises the role of services other than GUM in the management of STIs, and highlights the need to widen the scope of STI surveillance. However, the complex, expensive probability sampling technique used in NATSAL is not feasible for routine data collection. While not as accurate as population studies, primary care surveillance could provide estimates of the burden of morbidity within a group that closely corresponds to the general population. Almost $80 \%$ of the UK population consult a general practitioner at least once a year, and other primary care settings, such as family planning clinics are regularly attended by young people. ${ }^{8}$

A number of studies have provided information on the burden of STIs in primary care. ${ }^{9-11}$ One recent study, the Chlamydia Screening Studies (ClaSS) project, explored $C$ trachomatis prevalence in a general practice registered population aged 16-39 between 2001 and 2002, and included the collection of a limited range of variables for each patient. ${ }^{12}$ However, since these studies are unique they cannot be used for surveillance.

Infrequent panel surveys are a source of surveillance data. The Morbidity Statistics from General Practice (MSGP) was undertaken every 10 years and the last study, MSGP4, was undertaken in $1992 .{ }^{8}$ The dataset was derived from attendances over a 1 year period at 60 general practices in England and Wales, and represented a $1 \%$ population sample. Although not a random sample, the dataset is broadly representative of the general population in terms of age, sex, marital status, socioeconomic status, smoking behaviour, and burden of disease. ${ }^{8}$ However, although this is a potentially good source of data, it only gives an insight into the prevalence and determinants of incidence within the population at the time of the study. Nationally representative, prospective data are required to produce the timely insights into STI epidemiology that are needed to guide health policy.

Large prospective primary care surveillance datasets have become available over the past decade. The General Practice Research Database (GPRD) and MediPlus UK Primary Care Database are based on general practice attendances. The GPRD covers $6.4 \%$ of the UK population, is broadly similar to the Office for National Statistics (ONS) census in terms of age and sex, and includes data on diagnosis and treatment. MediPlus contains clinical and prescribing data from approximately 1.7 million patients who have attended 140 UK general practices since 1991 and is representative of the general population in terms of age, sex, and regional distribution. In addition, the Royal College of General Practitioners' General Practice Research Unit undertakes continuous sentinel surveillance in 72 practices covering 600000 people in England and Wales and collects demographic and diagnostic data. However, these datasets cannot be easily adapted to the specific requirements of STI surveillance because they do not capture data on reproductive history, contraceptive history, or sexual behaviour. Clinical samples are not taken, and the methodologies cannot be modified. In addition, the cost of using these datasets can be high. Information from family planning clinics is only consistently 
available from NHS family planning clinics and Brook Advisory Centres. The data, which are not validated and confined to contraceptive method, age, and sex of each patient at the first contact in the financial year, are reported to the Department of Health on the KT31 return.

The Public Health Laboratory Service (PHLS) laboratory reporting surveillance system managed by the Communicable Disease Surveillance Centre would be an alternative method of obtaining information on STIs diagnosed in primary care. The recent Department of Health (England) publication "Getting ahead of the curve, a strategy for combatting infectious diseases" recommended the mandatory collection of detailed data with all laboratory diagnoses and the PHLS system has been redeveloped to include more information on each report, such as ethnicity and source of report. ${ }^{13}$ This will potentially provide universal laboratory surveillance data for C trachomatis, Neisseria gonorrhoeae, HSV, and syphilis. Soon all positive and negative samples of $C$ trachomatis will be reported, which will allow the diagnostic rate to be estimated in a variety of clinical settings including primary care. Data from the roll out of the English chlamydia screening programme will also provide measures of screening uptake, coverage, and acceptability. However, both datasets are likely to be limited because of the restricted ability to collect relevant demographic and behavioural data. Ideally, surveillance should collect a range of information on factors that influence STI incidence (box 2), so that the diagnoses can be place within the context of the

Box 2 Factors that influence STI incidence

- sexual behaviour, such as number of lifetime sexual partners, frequency of sexual intercourse, and age at first sexual intercourse

- provision and access to healthcare services

- health service seeking behaviours

- contraceptive practice (lifetime), in particular condom use, and motivation for condom use

- reproductive history, such as termination of pregnancy, ectopic pregnancy, stillbirth, miscarriage

- cigarette smoking

- whether the patient has undergone instrumentation of the cervix, such as IUD insertion, dilatation, curettage, hysterosalpingography

- consulted for subfertility

- demographic characteristics, such as age, socioeconomic status, marital status

Table 1 Number of people required to detect differences in prevalence*

\begin{tabular}{|c|c|c|c|c|}
\hline \multirow{2}{*}{$\begin{array}{l}\text { Difference between } \\
\text { populations (\%) }\end{array}$} & \multicolumn{4}{|c|}{ Prevalence (\%) } \\
\hline & 2 & 5 & 10 & 20 \\
\hline-3 & - & 653 & 1421 & 2695 \\
\hline-2 & 482 & 1604 & 3312 & 6138 \\
\hline-1 & 2514 & 6943 & 13694 & 24840 \\
\hline+1 & 4023 & 8357 & 14950 & 25782 \\
\hline+2 & 1239 & 2311 & 3940 & 6609 \\
\hline+3 & 653 & 1125 & 1840 & 3009 \\
\hline
\end{tabular}

patient's reproductive and contraceptive history, sexual behaviour, demographic characteristics, and health seeking behaviour. Points of reference should also be included to allow comparisons with related studies.

Overall available primary care surveillance data are limited because many are either unique studies or collected once every 10 years; prospective studies are designed for general disease surveillance; little or no behavioural, demographic, and reproductive health data are collected; and many studies do not use standard diagnostic criteria. A new primary care STI surveillance system would help resolve these problems and create a resource to inform sexual health planning. Here we explore the attributes of such a system.

\section{REPEATED PANEL SURVEYS OF PRIMARY CARE ATTENDERS: A WAY FORWARD?}

The aims of the survey would be to estimate prevalence, and change in prevalence over time. The need for the timely collection of detailed data, together with a clinical sample suggests that a methodology based on a substantial population sample is inappropriate. A point prevalence sampling technique would increase efficiency and would be surprisingly small: a random sample of about 4000 for each sex would give a representative view of disease prevalence over the range $2 \%$ to $20 \%$ (table 1 ). The methodology and sample size calculations would have to be evaluated in a pilot study and the sampling strategy tailored to surveillance priorities, such as young people or geographic variation in prevalence. Patients could be invited to participate at clinic visits or by post. Measuring change in prevalence is an important method of evaluating intervention, but changes are difficult to predict and may be small. ${ }^{14}$ Again, a sample of 4000 would allow year on year trends to be detected accurately.

A self administered patient questionnaire that can be scanned directly into a computer database would be used to reduce data entry errors and allow a database to be constructed and "cleaned" within a few days. Information could thus be collected, interpreted, and disseminated quickly, and would allow surveillance to move towards the proactive stance required for health service planning.

Diagnosis would be the main outcome measure and tests and specimen types would need to be selected carefully. Molecular techniques, such as multiplex or microarray assays, could be used to diagnose several organisms, such as $C$ trachomatis, $N$ gonorrhoeae (including antibiotic resistance) using self obtained vaginal and vulval swabs or non-invasive specimens, such as urine. ${ }^{15}$ Mycoplasma genitalium, genital HSV infection, and human papillomavirus could also be included in the testing strategy but this may raise ethical concerns as screening for these infections is not generally recommended.

Potentially there are problems associated with a panel survey, the main concern being the comparability between the general practice age/sex register and the general population. Young men are less likely to be registered with a general practitioner than young women. For patients between the ages of 15 and 44 attending general practice the median contact days remain relatively constant over time at 6.4 per 100 people per week and 9.8/100 people per week, for males and females respectively. ${ }^{16}$ This panel survey technique is an idealised model and would have to be adapted to specific situations as it may not be feasible or appropriate to collect detailed data in some situations. In addition, the focus on general practitioners may make such a study difficult to implement at a time of increasing demands, although primary care research networks could be used as the framework for such an initiative. This system could also be used as the basis of a harmonised surveillance system that would allow a rapid evaluation of STI epidemiology and sexual health within and between countries.

\section{CONCLUSIONS}

The close relation between surveillance intelligence, disease prevention, and public health policy was highlighted by the Department of Health's "Getting ahead of the curve." At present, STI surveillance is limited by the relative lack of information from primary care: initiatives capable of identifying and evaluating emerging health care need to be 
developed. The method discussed here could allow an efficient assessment of STI prevalence, sexual health, and health service access behaviour. Such developments will be invaluable to future primary care STI surveillance, but no single method can effectively undertake surveillance. Other opportunities are evolving such as NHS electronic health records, a longitudinal record of patient health and health care, developments that have to be evaluated and assimilated into STI surveillance to complement existing knowledge. Creativity, innovation, and vision will be needed but it is only by doing this that we will be able to get ahead and stay ahead of the curve.

Sex Transm Infect 2003;79:174-176

\section{Authors' affiliations}

I Simms, A-K Hurtig, G Hughes, K A Fenton,

PHLS, Communicable Disease Surveillance

Centre, 61 Colindale Avenue, London

NW9 5EQ, UK

P A Rogers, PHLS Statistics Unit
Correspondence to: lan Simms, PHLS, Communicable Disease Surveillance Centre, 61 Colindale Avenue, London NW9 5EQ, UK; isimms@phls.org.uk

\section{REFERENCES}

1 Department of Health. The national strategy for sexual health and HIV. London: DoH, 2001.

2 Mårdh P-A, Wahren H. Klamydiainfektionen sexuellt överförd sjukdom i skuggan av aids. Läkartidningen 1990:87:3064-5.

3 Simms I, Nicoll A. Sexual health in England: a guide to national and local surveillance and monitoring data. London: Health Education Authority, 2000

4 Catchpole M, Harris J, Renton A, et al. Surveillance of sexually transmitted infections: fit for purpose? Int J STD AIDS 1999;10:493-4.

5 Simms I, Rogers $P$, Charlett A. The rate of diagnosis of pelvic inflammatory disease in general practice: England and Wales. Int $J$ STD AIDS 1999; 10:448-51.

6 Simms I, Hopwood J, Mallinson $\mathrm{H}$, et al. Changing screening strategies for genital chlamydia in family planning clinics: a good public health strategy? Eur J Contracept Reprod Health Care 2000;5:91-5.

7 Fenton KA, Korovessis C, Johnson AM, et al. Sexual behaviour in Britain: reported sexually transmitted infections and prevalent genital
Chlamydia trachomatis infection. Lancet 2001;358:1851-4.

8 OPCS. Morbidity statistics from general practice: fourth national study, 1991-1992 London: HMSO. 1995.

9 Stokes T. Screening for chlamydia in general practice: a literature review and summary of the evidence. J Public Health Med 1997; 19:222-32.

10 Simms I, Catchpole M, Brugha R, et al. Epidemiology of genital Chlamydia trachomatis in England and Wales. Genitourin Med 1996;73:122-6.

11 Department of Health. Chlamydia trachomatis. Summary and conclusions of trachomatis. Summary and conclusions of 1998.

12 The ClaSs Study Group. Evidence is not (yet) enough for evidence based policy of chlamydia screening. BM 2002:322:364-5.

13 Department of Health. Getting ahead of the curve: a strategy for combating infectious diseases. London: DoH, 2002.

14 Scholes D, Stergachis A, Heidrich F, et al. Prevention of pelvic inflammatory disease by screening for cervical chlamydial infection. $N$ Engl J Med 1996;334:1362-6.

15 Black CM. Current methods of laboratory diagnosis of Chlamydia trachomatis infections. Clin Microbiol Rev 1997; 10:160-84.

16 Royal College of General Practitioners, Birmingham Research Unit. The weekly returns service annual report. Birmingham: RCGP, 2000.

\section{New drugs for treating drug resistant HIV-1}

\section{A Isaac, D Pillay}

\section{Clinical management of virological failure remains an important and difficult issue for HIV physicians}

O ne of the major barriers to successful treatment of HIV-1 infection is the emergence of drug resistant virus. ${ }^{1}$ The greatest impact of resistance is that it limits the effectiveness of subsequent antiretroviral combinations following initial drug failure. At a population level, more than $50 \%$ of patients who fail therapy do so with viruses resistant to drugs within at least one class of drug, with 15-20\% with resistance to drugs within all three currently available classes (Health Protection Agency, unpublished data).
Therefore, there is an urgent requirement for new drugs with activity against such resistant species. Over the past year or so, there has been a welcome upsurge in data presented on new drugs, both within existing classes and new classes, with the promise of more effective therapies for HIV resistant viruses (see table 1).

\section{NUCLEOSIDE/NUCLEOTIDE ANALOGUES}

Nucleoside analogue drugs have been the mainstay of HIV therapy since zidovudine was first licensed in $1988,{ }^{2}$ and it is not surprising that resistance to this class of drugs is most common at a population level (Health Protection Agency, unpublished data). Despite some specific signature mutations for individual nucleoside analogues, there is increasing evidence for cross resistance

\begin{tabular}{|c|c|c|}
\hline Drug class & Stage of development & Comments \\
\hline \multicolumn{3}{|l|}{ NRTIs: } \\
\hline Tenofovir & Approved & Active against TAMs"1 \\
\hline Amdoxovir (DAPD) & Phase I/II & Active against various NRTI associated mutations, but not necessarily multidrug resistance ${ }^{78}$ \\
\hline Alovudine & Phase I/II & Activity against TAMs and antagonism with AZT and $d 4 \mathrm{~T}^{45}$ \\
\hline \multicolumn{3}{|r|}{ 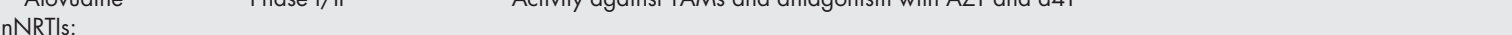 } \\
\hline TMC 120 & Phase I/II & Second generation nNRTIs active against nNRTI resistant viruses ${ }^{13} 14$ \\
\hline TMC125 & Phase I/II & \\
\hline Capravirine & Phase I/II & Activity against $\mathrm{K} 103 \mathrm{~N} / \mathrm{V} 106 / \mathrm{L} 1001$ mutants. Mutations at 181 codon confer high level resistance ${ }^{15}$ \\
\hline \multicolumn{3}{|r|}{ 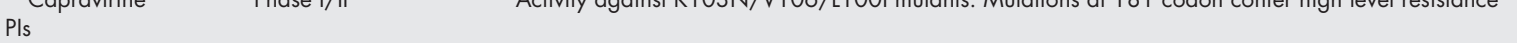 } \\
\hline Atazanavir & Phase III & $\begin{array}{l}\text { Favourable resistance profile in PI naive, but unclear cross resistance pattern in PI experienced. Good } \\
\text { side effects profiles }{ }^{16}\end{array}$ \\
\hline Tipranavir & Phase I/II & Likely to be effective in patients with experience of multiple protease inhibitors ${ }^{19}$ \\
\hline \multicolumn{3}{|r|}{ 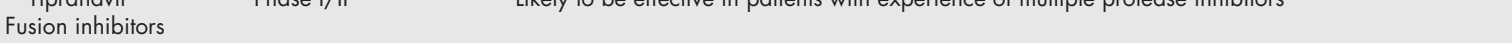 } \\
\hline Enfuvirtide (T-20) & Phase III & Active against multidrug resistant viruses ${ }^{20} 21$ \\
\hline
\end{tabular}


between certain drugs, such as ZDV and $\mathrm{d} 4 \mathrm{~T}$, as well as the emergence of mutations conferring broad cross resistance, such as the 69 insertions, and the Q151M constellation of mutations within reverse transcriptase. Interesting data have been presented for alovudine, a thymidine analogue previously shown to have considerable toxicity in the clinic. Now reassessed at lower doses, activity is observed in patients with ZDV/d4T resistance (up to five thymidine analogue resistance associated mutations, TAMS) although antagonism between these thymidine analogues is observed when used in combination. ${ }^{34}$ More data are awaited for this rejuvenated compound. Amdoxovir (DAPD) is a new nucleoside analogue prodrug whose oral administration leads to a rapid in vivo conversion to $(-)$ - $\alpha$-D-dioxalane guanosine (DXG). Resistance to this drug in the laboratory appears to involve the K65R and L74V mutations, similar to those observed for abacavir (although ABC failure is rarely associated with these mutations in the clinic). ${ }^{5}$ Phase I/II studies demonstrate a reasonable activity of this drug against nucleoside analogue resistant viruses, although more data are needed before clarifying its potential role. However, activity in vitro is compromised by the multinucleoside resistance mutation Q151M together with changes at amino acid 69 of reverse transcriptase, which may limit its role in higher nucleoside analogue experienced patients. ${ }^{67}$ The drug attracting most excitement at present is the recently approved nucleotide analogue tenofovir, which appears to be unencumbered by the toxicity problems of its cousin, adefovir. As for many other drugs, the HIV mutations in reverse transcriptase associated with reduced activity in the clinic are not necessarily those selected by tenofovir in the laboratory (K65R). This is because the drug has been most widely tested in drug experienced patients in whom resistant virus already exists and predictors of poor response can be identified. Thus, common nucleoside analogue resistance mutations such as M41L, L210W (possibly a key marker in this respect), and $\mathrm{T} 215 \mathrm{Y}$ appear to reduce, although not negate, clinical efficacy; nevertheless, the widespread use of tenofovir in salvage therapy and promising first line treatment trial data suggest that it represents an important addition to our antiviral armoury. ${ }^{8-10}$

\section{NON-NUCLEOSIDE REVERSE TRANSCRIPTASE INHIBITORS}

The phenomenon of extensive cross resistance between nNRTIs is one of the more widely accepted truths of HIV drug resistance, owing to the small binding site for this group of drugs within the viral reverse transcriptase. ${ }^{11}$ The key mutations in this regard are K103N, T181C, and G190A/E, all of which compromise nevirapine, efavirenz, and delavirdine responses, and this cross resistance represents a major limitation of the class as a whole. However, two new compounds, TMC 125 and TMC120, appear to have activity against such resistance viruses, both in vitro and in vivo. ${ }^{12}$ Another compound (capravirine) demonstrated activity against a virus bearing the K103N or V106A or Li00I single mutation, although high level resistance to this drug was reported in the presence of mutations at codon $181 .{ }^{14}$ It appears not so much that different patterns of resistance mutations are observed with these new nNRTI drugs, but rather that emergence of resistance is much slower than existing nNRTIs - note that single dose nevirapine in pregnancy is sufficient to select for resistant mutantsand that the well recognised nNRTI mutations have a marginal, and possibly clinically irrelevant, impact on fold susceptibility. It is argued that these properties are a function of the unique structures of these second generation nNRTIs, in the context of binding to the RT enzyme. We look forward to more extensive clinical trial data for both these drugs.

\section{PROTEASE INHIBITORS}

Issues of resistance and cross resistance are particularly pertinent to the protease inhibitor class of drugs. Many claims have been made on the apparent uniqueness of resistance patterns for specific drugs, based on in vitro data, which do not then translate into clinical benefit for that drug in PI experienced patients. Two new PIs have now undergone initial clinical evaluation. Atazanavir (ATZ), soon to be available within an expanded access programme, demonstrated different resistance profiles when used in PI naive or PI experienced patients. In the former group, resistance emerges with the I50L and A7IV mutations. ${ }^{15}$ This is a unique combination since amprenavir resistance mutations include a different amino acid change at position 50 (namely, I50V), although the A7IV mutation is a polymorphism (not infrequently observed in the absence of PI therapy). By contrast, in PI experienced patients, some level of cross resistance between atazanavir and other PIs was apparent. ${ }^{16}$ and therefore the utility of this drug as a second line PI may be limited. Clinical data for this scenario are awaited at the time of writing. Clinical data have also been presented for tipranavir, which shows potency against viruses containing a large variety of PI resistant mutants in vitro. ${ }^{17}$ Clinical activity was indeed observed in PI experienced patients, with a suggestion that a very large number of PI resistant mutations were required to compromise activity. ${ }^{18}$ More work is required to further clarify such "clinical cut offs" whereby clinicians can be guided on the likely effect of this new drug in a patient with existing PI resistant virus.

\section{FUSION INHIBITORS}

Data are now emerging from the trials of T-20 (enfuvirtide), the first fusion inhibitor to enter the clinic. Since the phase III trials were undertaken in heavily pretreated patients it is not surprising that failure rates (lack of full suppression) were relatively high overall; however, this affords the opportunity to characterise the emergence of resistance. ${ }^{19} 20$ Data from phase II studies demonstrate that the majority of such failure patients had mutations in the gp4l region targeted by the drug-namely, between amino acids $36-45$, which indeed confirms that activity of the drug is mediated through the proposed mechanism. ${ }^{21}$ Since variation in this region is very rare in T-20 naive patients, including those infected with non-subtype B viruses, it can be assumed that previous RT inhibitor and PI therapy will not compromise T-20 activity itself. ${ }^{22}$ The key issue with use of T-20 in salvage therapy will therefore be the choice of other active drugs to combine with it. Of interest, the second generation fusion inhibitor T-1249 appears to be active against most $\mathrm{T}-20$ resistance mutants, although this is based on in vitro evidence alone. ${ }^{23}$

\section{CONCLUSION}

Despite the undoubted success of antiretroviral therapy clinical management of virological failure remains an important and difficult issue for HIV physicians. Since such patients often have drug resistant virus, the choice of new combinations is often based, at least to some extent, on our knowledge of resistance characteristics of available drugs. We have summarised the data on a whole series of new drugs within existing and new classes. After some years of promising in vitro data, these drugs have demonstrated promise in clinical trials, with particular interest focused on unique resistance patterns, or the slow development of resistance. As further clinical trial data are presented for new drugs, it is important for HIV physicians to ask two specific questions. Firstly, what are the resistance patterns at baseline, which define success or failure of this new drug in antiretroviral experienced patients and, secondly, what are the resistance correlates of failure when used as a first line drug? It is answers to these questions that will contribute to identifying the optimal role of these promising new drugs in routine clinical practice.

Sex Transm Infect 2003;79:176-178 


\section{Authors' affiliations}

A Isaac, Whittal Street Clinic, Birmingham, UK D Pillay, PHLS Antiviral Susceptibility Reference Unit, Birmingham, UK

Correspondence to: Dr Deenan Pillay, Department of Virology, Windeyer Institute, University College Hospital, 46 Cleveland Street, London WIT 4JF, UK; d.pillay@ucl.ac.uk

Accepted for publication 27 February 2003

\section{REFERENCES}

1 Pillay D, Taylor S, Richman DD. Incidence and impact of resistance against approved antiretroviral drugs. Rev Med Virol 2000;10:231-53.

2 Fischl MA, Richman DD, Grieco MH, et al The efficacy of azidothymidine (AZT) in the treatment of patients with AIDS and AIDS-related complex: a double blind placebo-controlled trial. N Eng J Med 1987;317:185-97.

3 Calvez V, Tubiana R, Ghosen J, et al. MIC-3 10 reduced markedly viral load in patients with virological failure despite multiple drug therapy: result from a 4-week phase II study. Antiviral Therapy

2002;7(suppl 1):S5

4 Vang L, Zhang $H$, Palmer $S$, et al. In vitro effects of MIV-310 (Alovudine, 3-fluorodeoxythymidine, FLT) against HIV mutants. Antiviral Therapy 2002;7(suppl 1):S25.

5 Mewshaw J, Myrick FT, Wakefield DA, et al. Dioxolane guanosine, the active form of the prodrug diaminopurine dioxolane, is a potent inhibitor of drug-resistant HIV-1 isolates from patients for whom standard nucleoside therapy fails. J Acquir Immune Defic Syndr 2002;29:11-20.

6 Eron J, Kessler H, Thompson M, et al. Clinica HIV suppression after short-term monotherapy with DAPD. 40th Interscience Conference on Antimicrobial Agents and Chemotherapy, Toronto, abstract 690, 2000.

7 Jeffrey J, et al. Amdoxovir, a nucleoside reverse transcriptase inhibitor, is active against HIV mutants resistant to a standard nucleoside therapy. Antiviral Therapy 2001;6(supp 1):10-11.
8 Squires K, Pierone G, Berger D, et al. Tenofovir DF: a 48-week final analysis from phase III randomised, double blind placebo controlled study in antiretroviral experienced patients. Ninth Conference on Retroviruses and Opportunistic Infections, Seattle, abstract 413-W, 2002

9 Staszewski S, Gallant J, Pozniak A, et al. Efficacy and safety of tenofovir disoproxil fumarate (TDF) versus stavudine (d4T) when used in combination with lamivudine (3TC) and efavirenz (EFV) in HIV-1 infected patients naive to antiretroviral therapy (ART): 48-week interim results. Fourteenth International AIDS Conference, Barcelona, abstract 17, 2002

10 Miller MD, Margot NA, Cheng AK, et al. Expanded response analysis of Tenofovir DF therapy by base line resistance genotype and phenotype. Fourteenth International AIDS Conference, Barcelona, abstract Th Or B1390, 2002

11 Richman DD, Havlir D, Corbeil J, et al. Nevirapine resistance mutations of human immunodeficiency virus type 1 selected during therapy. J Virol 1994;68:1660-66.

12 Gruzdev B, Rakhmanova A, Van t'Klooster G, et al. One week of monotherapy with TMC-125, a novel highly potent NNRTI, produces a mean 2 log reduction in viral load in antiretroviral naive HIV-1 infected volunteers. Eighth European Conference on Clinical Aspects and Treatment of HIV Infection, Athens, abstract 09, 2001.

13 Gazzard B, Pozniak A, Arasteh K, et al. TMC 125, a next-generation NNRTI demonstrates high potency after 7 days therapy in treatment-experienced HIV-1-infected individuals with phenotypic NNRTI resistance. Ninth Conference on Retroviruses and Opportunistic Infections, Seattle, abstract 5, 2002.

14 Potts KE, Fujiwara T, Sato A, et al. Antiviral activity and resistance profile of AG-1549, a novel HIV-1 non-nucleoside reverses transcriptase inhibitor. Sixth Conference on Retroviruses and Opportunistic Infections, Chicago, 1999, abstract 12

15 Colonno RI, Friborg J, Rose RE, et al. Identification of amino acid substitutions correlated with atazanavir susceptibility in patients treated with atazanavir-containing regimens. Antiviral Therapy 2002;7(suppl 1): $\$ 56$
16 Robinson B, Riccardi K, Gong Y, et al. BMS-232632, a highly potent human immunodeficiency virus protease inhibitor that can be used in combination with other available antiretroviral agents. Antimicrob Agents Chemother 2000;44:2093-9

17 Larder BA, Hertogs K, Bloor S. Tipranavir inhibits broadly protease inhibitor-resistant HIV-1 clinical samples. AIDS 2000; 14: 1943-8.

18 Schwartz R, Kazanjian P, Slater L, et al. Resistance to tipranavir is uncommon in a randomized trial of tipranavir/ritonavir (TPV/RTV) in multiple-Pl-failure patients (B 1 182.2). Ninth Conference on Retroviruses and Opportunistic Infections, abstract 562, 2002.

19 Henry K, Lalezari J, Ohearn M, et al. Enfuvirtide (T-20) in combination with an optimized background (OB) regimen vs $O B$ alone in patients with prior experience or resistance to each of the three classes of approved antiretrovirals in North America and Brazil (TORO 1). Fourteenth International AIDS Conference, Barcelona, abstract LbOr19B, 2002.

20 Clotet B, Lazzarin A, Cooper D, et a Enfuvirtide (T-20) in combination with an optimized background $(O B)$ regimen vs $O B$ alone in patients with prior experience or resistance to each of the three classes of approved antiretrovirals in Europe and Australia (TORO 2). Fourteenth International AIDS Conference, Barcelona, abstract LbOr19A, 2002.

21 Greenberg ML, Sista P, Miralles GD, et al. Characterization of baseline and treatment-emergent resistance to T-20 (enfuvirtide) observed in Phase II clinical trials: substitutions in gp4 1 amino acids 36-45 and enfuvirtide susceptibility of virus isolates. Antiviral Therapy 2002;7(Suppl 1):S16.

22 Xu L, Hue S, Pillay D, et al. Minimal variation in T-20 binding domain of different HIV-1 subtypes from antiretroviral naïve and experienced patients. AIDS 2002;16:1684-6.

23 Greenberg $M L$, Davison D, Jin L, et al. In vitro antiviral activity of T-1249: a second generation fusion inhibitor. Antiviral Therapy 2002;7(Suppl 1):S10. 